\title{
STELLAR AND TOTAL MASS IN EARLY-TYPE LENSING GALAXIES
}

\author{
IGNACIO FERRERAS ${ }^{1}$, Prasenjit SAHA ${ }^{2}$, AND Liliya L.R. WiLliams ${ }^{3}$ \\ ${ }^{1}$ Department of Physics and Astronomy, University College London, Gower St. London WC1E 6BT, England \\ 2 Astronomy Unit, Queen Mary and Westfield College, University of London, London E1 4NS, England \\ ${ }^{3}$ Dept. of Astronomy, University of Minnesota, 116 Church Street SE, Minneapolis MN 55455
}

Accepted for publication in the Astrophysical Journal Letters

\begin{abstract}
For 18 well-observed gravitationally lensed QSOs, we compare new non-parametric mass profiles for the lensing galaxies with stellar-population models derived from published HST photometry. The large volume of parameter space searched - with respect to the possible star formation histories - allows us to infer robust estimates and uncertainties for the stellar masses. The most interesting results are: (1) the transition from little or no dark matter in the inner regions $\left(\lessgtr r_{e}\right)$ to dark matter dominating on the $\sim 5 r_{e}$ scale $(\sim 20 \mathrm{kpc})$ is clearly seen in massive ellipticals; (2) Such a trend is not seen in lower-mass galaxies, so that the stellar content dominates the mass budget out to $\sim 5 r_{e}$; (3) the radial gradient in the dark-matter fraction for these intermediate redshift galaxies agrees with published data on nearby galaxies. This result can help reconcile the discrepancies found in recent estimates of dark matter in elliptical galaxies using different techniques (e.g. Planetary nebulae versus X-ray). The observed trend suggests the stellar component in massive galaxies extends further out in terms of the dark matter scale radius.
\end{abstract}

Subject headings: galaxies: evolution — galaxies: formation — galaxies: elliptical and lenticular, cD — dark matter

\section{INTRODUCTION}

For galaxies further than $\sim 100 \mathrm{Mpc}$, the usual kinematic tracers of galactic dynamics become increasingly difficult or impossible to use. But at larger distances, nature sometimes provides a very different indicator of galaxy mass-strong lensing of quasars. Galaxies with a quasar conveniently placed behind them, which they then lens into multiple images, are rare. But for those galaxies it is fairly easy to measure masses, even to $z \sim 1$. In recent years there has been progress on estimating the $M / L$ in samples of lensing galaxies (Keeton et al. 1998, Kochanek et al. 2000, Rusin et al. 2003). For a few systems there have been efforts to combine lensing and velocity dispersions (Treu \& Koopmans 2002a,b, Koopmans \& Treu 2003).

In this Letter we go beyond simple $M / L$ for a sample of lensing galaxies and try to recover the distribution of stellar and dark mass within galaxies. Our technical innovations are (i) we use observed colors to model the stellar population in detail and hence map the stellar mass, and (ii) we use the method of pixelated lens reconstruction to make detailed profiles of the total mass; we pay particular attention to quantifying the uncertainties in both departments.

Our sample comprises 17 early-type galaxies over a wide range of redshifts $(0.3<z<1)$ and a bulge $(z=0.04)$. The sample has been selected from the CASTLeS group database ${ }^{1}$. The main properties are listed in Table 1 . We assume a concordance cosmology $\left(\Omega_{\mathrm{m}}=0.3, \Omega_{\Lambda}=0.7\right)$ with $H_{0}^{-1}=14 \mathrm{Gyr}$.

\section{MEASURING LENSING MASSES}

For most lensed quasars, it is fairly easy to fit a model galaxy lens to the image positions and hence provide a map of the total sky-projected mass. But such a map, although a reasonable first approximation, will be non-unique because of lensing degeneracies (Falco et al. 1985, Gorenstein et al. 1988, Saha 2000). A way around this problem is to generate an ensemble of mod- els (Williams \& Saha 2000, Trotter et al. 2000, Keeton \& Winn 2003) all models constrained to reproduce the observed image positions (and time delays if known) precisely. From the model ensemble, estimates and uncertainties of any desired quantityfor example, the mass at a given projected radius - can be easily extracted. We use the PixeLens code (Saha \& Williams 2004) which more or less automates the whole procedure, even for complex lenses like B1608+656.

The model-ensemble technique (we used 200 models per lens) makes our mass uncertainties larger than in previous work on $M / L$ ratios, but much more realistic. Four-image lenses tend to be better constrained than two-image lenses. Also, systems with known time delays allow much tighter mass estimates (provided $H_{0}$ is assumed, as we do here).

\section{MEASURING STELLAR MASSES}

The stellar mass content can be determined from the photometry although a fair share of assumptions must be invoked in order to transform light into mass. These assumptions relate to the age, metallicity and mass distribution of the unresolved stellar populations. The latter can be reduced to a time-independent, universal initial mass function (IMF) as suggested by observations (see e.g. Wyse 1998). In this paper we explore two IMFs: Salpeter (1955) and Chabrier (2003), both defined between $0.1 M_{\odot}$ and $100 M_{\odot}$. The former assumes a simple power law over the allowed range of stellar masses. The IMF proposed by Chabrier (2003) has a very similar upper-mass dependence as the Salpeter function. However, the low-mass end assumes a flatter - more physical - behaviour, following a lognormal distribution. This IMF gives $M / L$ ratios which are a factor $\sim 1.5$ smaller than those for a Salpeter IMF.

The main difference between most of the IMFs proposed (e.g. Salpeter 1955; Miller \& Scalo 1979; Kroupa, Tout \& Gilmore 1993; Chabrier 2003) lies in the relative contribution from stars with masses $M<M_{\odot}$. Since these stars have a large

${ }^{1}$ See the CASTLeS page, http: //cfa-www. harvard.edu/glensdata/for a list. 

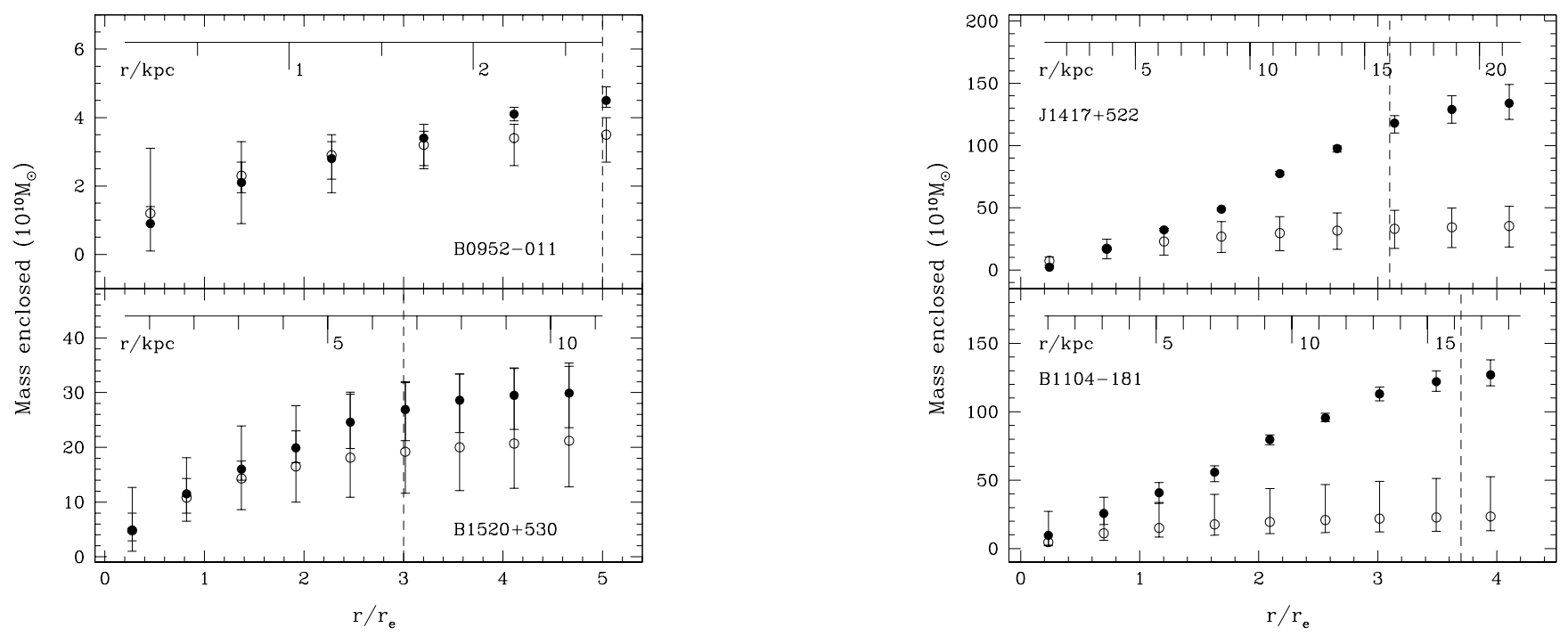

Fig. 1.- Profiles of the mass enclosed as a function of radius for two low-mass (left) and two massive early-type galaxies (right). The solid and empty circles give the total and stellar mass content, respectively. The stellar masses assume a Chabrier IMF but a Salpeter IMF leads to qualitatively the same result. The error bars indicate $90 \%$ confidence intervals. The vertical dashed line gives the position of $r_{\text {lens }}$.

$M / L$ ratio, stellar populations born with either IMF feature very similar spectral energy distributions, albeit with a different normalization for a given stellar mass (see e.g., Bruzual \& Charlot 2003). Therefore, a photo-spectroscopic analysis cannot be used reliably to constrain the IMF at the low-mass end. Instead, we will use the available photometry from our sample and compute the stellar mass content corresponding to either a Salpeter or a Chabrier IMF. These two mass distributions represent a robust range of possible values between the unphysically high $M / L$ imposed by a simple power law extrapolated to low masses and a more realistic distribution.

The age and metallicity distribution can be constrained by a comparison of the photometry with a simple model of star formation. We assume a 3-parameter model which reduces the description of the stellar populations to a single metallicity $\left(Z_{\star}\right)$, a formation epoch $\left(t_{\mathrm{FOR}}\right)$, and a formation timescale $\left(\tau_{\mathrm{SF}}\right)$, so that at any given time the star formation rate is $\psi(t) \propto$ $\exp \left(-\Delta t / \tau_{\mathrm{SF}}\right)$, with $\Delta t=t-t_{\mathrm{FOR}}$. Each choice of parameters $\left(Z_{\star}, t_{\mathrm{FOR}}, \tau_{\mathrm{SF}}\right)$ represents a possible formation scenario, and can be convolved with simple stellar populations (SSP) in order to generate a composite model from which various photospectroscopic observables can be retrieved. We use the Bruzual $\&$ Charlot (2003) population synthesis models.

We use the published photometry of the lenses from the CASTLeS group (Rusin et al. 2003) which was obtained from HST/WFPC2+NICMOS images after carefully subtracting the contribution from the sources. Each set of measurements correspond to a number of colours (from 1 to 5 ) which is used in order to constrain the parameter space described above. We use their radial fits to generate the profile of the stellar component. Galactic reddening was included in the analysis by using the $E(B-V)$ values from Rusin et al. (2003) and applying a dust correction according to the $\mathrm{R}=3.1$ curve of Fitzpatrick (1999).

The best fit for each lens was obtained using an adaptive grid in the 3-dimensional parameter space that describes all possible star formation histories in our model, and using a Metropolis algorithm (see e.g., Binney et al. 1992, or Saha 2003) to find the uncertainties which are quoted with respect to the 5th-95th percentiles ( $90 \%$ confidence interval) throughout the paper. The stellar mass content is computed for the best fit, with respect to the light inside the half-light radius $\left(r_{e}\right)$.

\section{STELLAR VS TOTAL MASS}

Figure 1 shows the mass profiles (stellar and total) for four galaxies, two with $M_{t o t} \ll 10^{12} M_{\odot}$ (left) and two with $M_{t o t} \gtrsim$ $10^{12} M_{\odot}$ (right). The result is striking: the low-mass galaxies have little or no dark matter at all observed radii. The highmass galaxies have little or no dark matter inside of $r \sim r_{e}$, but at large radii they are dominated by dark matter. Here we have a clear indication that massive galaxies have a gradient in their dark-matter fraction.

Rather than presenting mass profiles for all the galaxies in our sample, we will summarize the profiles in two ways: first by comparing stellar and total mass at a chosen radius, and then by quantifying the dark-matter gradient.

In Figure 2 and Table 1 we show three derived aperture masses: the total mass derived from lensing, the stellar mass derived assuming a Salpeter IMF, and the stellar mass derived assuming a Chabrier IMF. Uncertainties are at $90 \%$ confidence. The aperture radius in all cases is a quantity we call $r_{\text {lens }}$, and it is the largest radius at which the lensing mass is wellconstrained. In general, $r_{\text {lens }}$ represents the radius over which there is lensing information, and is roughly the radius of the outermost image. From Figure 2 it is apparent that the darkmatter fraction increases with mass, and the trend is compatible with the observed tilt of the NIR fundamental plane (Mobasher et al. 1999; Ferreras \& Silk 2000). Additionally, we see that $M_{s t}$ is estimated as more than $M_{t o t}$ in a few cases for the Salpeter IMF, but never for the Chabrier IMF; while it would be cavalier to argue against a Salpeter IMF on this basis, it does suggest that future comparisons of lensing and stellar-population models may help constrain the IMF.

We now consider the gradient in the dark-matter fraction. 


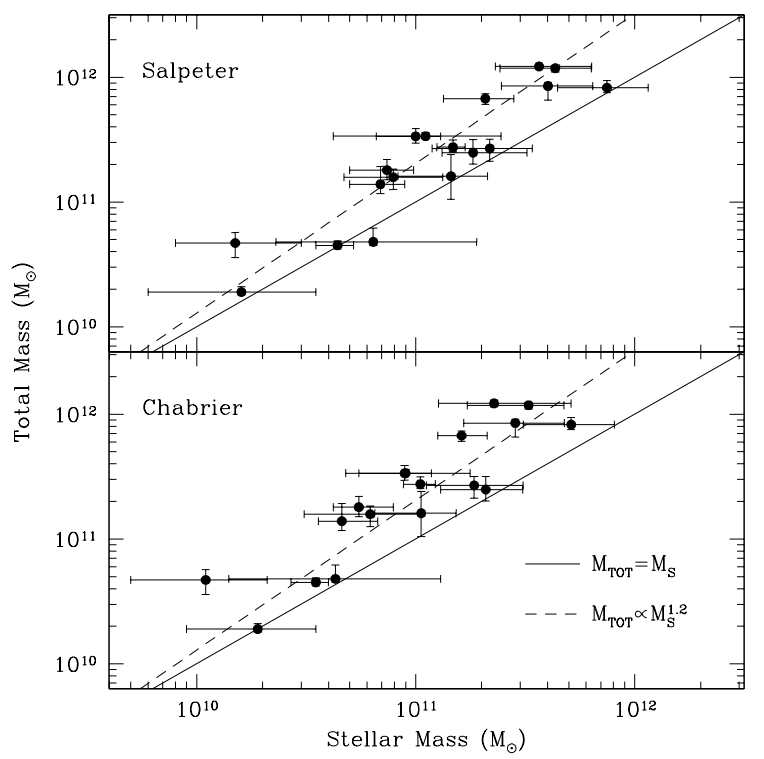

FIG. 2.- A comparison of total and stellar masses for our sample of gravitational lens early-type galaxies (all measured inside $\left.r_{\text {lens }}\right)$. The upper and lower panels correspond to a Salpeter and a Chabrier IMF, respectively. The solid line represents $M_{t o t} / M_{s t}$ and the dashed line follows the expected correlation from the tilt of the fundamental plane for ellipticals.

Following Napolitano et al. (2005; hereafter N05) we introduce

$$
\nabla \Upsilon \equiv \frac{r_{e}}{\Delta r}\left[\left(\frac{M_{t o t}}{M_{s t}}\right)_{\text {out }}-\left(\frac{M_{t o t}}{M_{s t}}\right)_{\text {in }}\right] .
$$

Even though $\nabla \Upsilon$ is a noisy quantity, it has advantages over $M_{\text {tot }} / M_{s t}$. Being a gradient it is not sensitive to the choice of wavebands used to measure stellar luminosity. Also, $\nabla \Upsilon$ is unlikely to be very sensitive to the redshift evolution of galaxies, because to first order $M_{t o t} / M_{s t}$ will change at the same rate throughout the inner parts of a galaxy as its stellar population ages. We are able to compute $\nabla \Upsilon$ with tolerable uncertainty for only a subsample of galaxies.

The work of N05 differs from ours in several respects: (i) they derived masses from the kinematics of planetary nebulae and globular clusters; (ii) their galaxies are nearby whereas ours span range from $z \simeq 0.3$ to and $z \simeq 1$; (iii) they used threedimensional radial gradients while we are working with projected quantities. Point (ii) is unlikely to affect $\nabla \Upsilon$ much, as we have already argued. Regarding point (iii), the difference is minimal for the estimation of the stellar masses, since as Figure 1 shows, the stellar mass is concentrated within the central few kpc; even for the more extended distribution of the dark matter, most of the mass that makes up the total line of sight mass resides at small radii. In general, 2D and 3D $\nabla \Upsilon$ estimates differ by less than $50 \%$. We conclude that we can directly compare with N05.

Figure 3 compares our estimates of $\nabla \Upsilon$ with those of N05 in the format of their Fig. 7. There is a general agreement between the two sets of results, at least over the mass range covered by both data sets. Our values are somewhat higher for $11<\log \left(M_{\star} / M_{\odot}\right)<11.5$ range, but with the current small

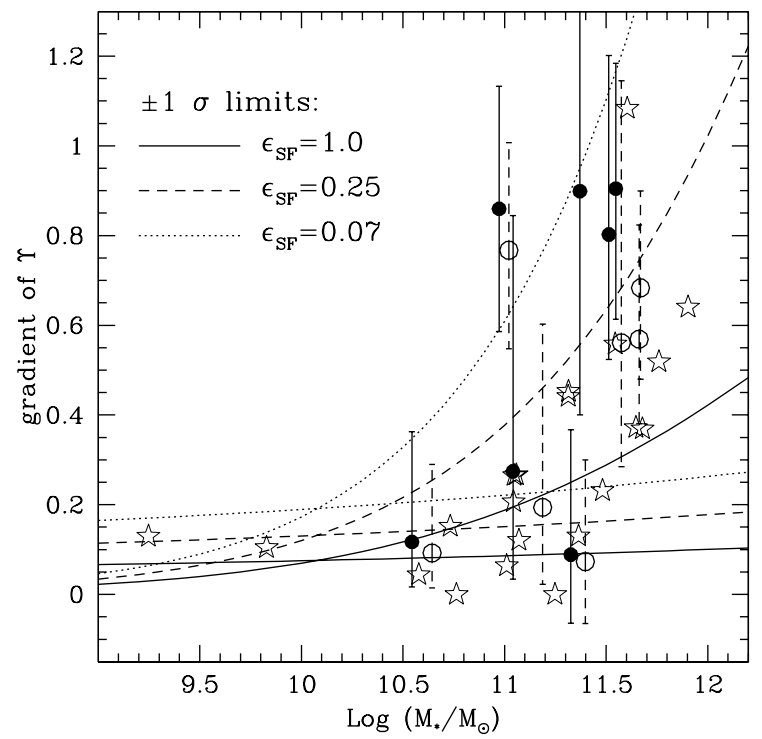

FIG. 3.-Dark matter gradient $\nabla \Upsilon$, computed between $r_{\text {in }} \simeq$ $0.25 r_{e}$ and $r_{\text {out }} \simeq 4.5 r_{e}$. Filled circles correspond to the Chabrier IMF and empty circles to the Salpeter IMF. The error bars correspond roughly to $1 \sigma$. This figure may be compared with Fig. 7 of Napolitano et al. (2005). The star symbols are taken from that paper. The curves are $1 \sigma$ bands for model predictions with different star-formation efficiencies, also adapted from Napolitano et al.

numbers we cannot assess significance. In Figure 3 we have also plotted the predictions of $\Lambda \mathrm{CDM}$ models by N05. This requires some justification. First, the models were computed for the $r_{e}-M_{s t}$ relation characteristic of the N05 data set, but our $r_{e^{-}}$ $M_{s t}$ turns out to be very similar. Second, the models are based on NFW $\Lambda$ CDM predictions for halos at $z \approx 0$; do they still apply to our data, with $z \sim 0.3-1$ ? Fortunately, yes, because the relation between the concentration parameter and halo mass is fairly robust with respect to the redshift of observation in the $z=0-3$ range (see Fig. 14 of Wechsler et al. 2002). The curves in Figure 3 show three different star-formation efficiencies: $\epsilon_{S F}=0.07,0.25$, and 1 , where $\epsilon_{S F}$ is defined as the ratio between total stellar and baryonic matter. The general trend is that higher stellar mass systems should have a monotonically larger contribution from dark matter with increasing radius. This can be understood intuitively as follows (cf. section 3.4 of N05). In galaxies with higher stellar masses the stellar component extends to larger radii, in terms of NFW profile scale radius, and so larger radii are more dark matter dominated. Our data is consistent with that trend. However, one should be cautious when interpreting the results with respect to $\epsilon_{S F}$. The N05 models disregard the effect of the gas collapsing to the centre of the halo and its effect on the dark matter density profile.

\section{DISCUSSION}

In the Milky Way it is generally held that in the inner few kpc and on mass scales of $\sim 10^{11} M_{\odot}$ there is little or no dark matter, whereas on the scale of $\sim 100 \mathrm{kpc}$ and $\sim 10^{12} M_{\odot}$ dark matter completely dominates. In this Letter, we see the same transition in elliptical galaxies at redshifts of 0.3 to 1 , based on projected mass distribution derived from lensing. The dark matter 
TABLE 1

APERTURE MASS $\left(\times 10^{10} M_{\odot}\right)$

\begin{tabular}{|c|c|c|c|c|c|c|c|}
\hline Object & $\mathrm{z}$ & $\mathrm{r}_{e} / \mathrm{kpc}$ & $\mathrm{r}_{\text {lens }} / \mathrm{kpc}$ & $M_{V}$ & $\mathrm{M}_{\text {tot }}\left(<r_{\text {lens }}\right)$ & $\mathrm{M}_{\text {salp }}\left(<r_{\text {lens }}\right)$ & $\mathrm{M}_{\text {chab }}\left(<r_{\text {lens }}\right)$ \\
\hline B0047-280 & 0.49 & 5.4 & 8.6 & -22.5 & $33.7_{32.3}^{36.2}$ & $11.1_{4.2}^{24.5}$ & $9.0_{4.8}^{17.7}$ \\
\hline В0142-100 & 0.49 & 3.1 & 5.6 & -22.7 & $24.9_{202}^{31.7}$ & $18.3_{132}^{32.2}$ & $20.9_{130}^{30.8}$ \\
\hline J0414+053 & 0.96 & 14.5 & 29.0 & -23.1 & $82.5_{75.3}^{93.9}$ & $74.7_{44.5}^{115.4}$ & $51.3_{31.0}^{80.8}$ \\
\hline B $0818+122$ & 0.39 & 4.8 & 10.6 & -22.0 & $67.4_{60.7}^{73.6}$ & $20.8_{13.4}^{28.1}$ & $16.2_{12.6}^{21.2}$ \\
\hline $\mathrm{J} 0951+263$ & 0.20 & 0.7 & 2.1 & -19.3 & $4.7_{3.6}^{5.7}$ & $1.5_{0.8}^{3.0}$ & $1.1_{0.5}^{2.1}$ \\
\hline В0952-011 & 0.38 & 0.5 & 2.5 & -19.4 & $4.5_{4.2}^{4.9}$ & $4.4_{3.5}^{5.2}$ & $3.5_{2.7}^{4.0}$ \\
\hline B1009-025 & 0.78 & 1.6 & 4.8 & -21.0 & $18.0_{151}^{22.0}$ & $7.4_{50}^{9.8}$ & $5.5_{42}^{7.9}$ \\
\hline J1017-204 & 0.86 & 2.4 & 2.4 & -21.6 & $4.8_{4.5}^{6.2}$ & $6.4_{2.3}^{19.0}$ & $4.3_{1.4}^{13.0}$ \\
\hline B1030+074 & 0.60 & 2.6 & 4.7 & -21.8 & $16.1_{105}^{24.1}$ & $14.5_{8.3}^{21.3}$ & $10.6_{65}^{15.3}$ \\
\hline B1104-181 & 0.73 & 4.4 & 16.3 & -22.7 & $122.0_{115.0}^{130.0}$ & $36.6_{23.1}^{63.7}$ & $22.8_{12.7}^{51.2}$ \\
\hline В1115+080 & 0.31 & 2.3 & 7.4 & -21.1 & $27.5_{254}^{31.5}$ & $14.8_{125}^{16.8}$ & $10.5_{8.8}^{12.3}$ \\
\hline $\mathrm{J} 1411+521$ & 0.46 & 2.9 & 9.3 & -21.2 & $33.6_{29.6}^{38.8}$ & $10.0_{6.6}^{13.0}$ & $8.9_{5.5}^{11.8}$ \\
\hline $\mathrm{J} 1417+522$ & 0.81 & 5.2 & 16.1 & -23.4 & $118.0_{110.0}^{124.0}$ & $43.4_{24.3}^{63.4}$ & $32.8_{17.2}^{47.6}$ \\
\hline $\mathrm{B} 1422+231$ & 0.34 & 1.5 & 6.0 & -20.7 & $\begin{array}{l}15.8_{126}^{18.4} \\
\end{array}$ & $7.9_{4.7}^{13.3}$ & $6.2_{3}^{10.6}$ \\
\hline В1520+530 & 0.72 & 2.2 & 6.6 & -22.4 & $26.9_{21.2}^{31.8}$ & $21.8_{11.9}^{34.1}$ & $18.5_{11.2}^{30.9}$ \\
\hline $\mathrm{B} 1608+656$ & 0.63 & 4.2 & 6.3 & -23.1 & $85.0_{65.9}^{90.9}$ & $40.2_{246}^{64.3}$ & $28.5_{166}^{47.8}$ \\
\hline В2149-274 & 0.50 & 2.9 & 4.1 & -21.9 & $13.9_{11.7}^{19.3}$ & $6.9_{5.0}^{8.9}$ & $4.6_{3.6}^{6.7}$ \\
\hline B2237+030 & 0.04 & 3.1 & 0.9 & -20.9 & $1.9_{1.8}^{2.1}$ & $1.6_{0.6}^{3.5}$ & $1.9_{0.9}^{3.5}$ \\
\hline
\end{tabular}

fraction tends to increase with the total mass. Incidentally we note that one of the possible mechanisms that can be invoked to explain the tilt of the fundamental plane involves such a trend between stellar and total mass with a very similar scaling behavior (Ferreras \& Silk 2000). Part of the tilt is explained by non-homology effects (Trujillo et al. 2004), but our data suggest a further contribution to the tilt from the different stellar vs dark matter distributions. We claim that most of the tilt of the fundamental plane - at least when the observables used to define it reach beyond $r_{e}$-is caused by the correlation between dark matter fraction and galaxy mass as presented in this Letter. The gradient of the dark matter fraction inside individual galaxies is also similar to what is inferred in nearby galaxies. These results indicate that the distribution of dark matter in galaxies has not changed much since $z=1$.

Enlarging the sample of galaxies for which stellar and dark- matter profiles can be reconstructed in this way is an obvious goal for future work. But another line of future work is to improve the spatial resolution in some cases. A possible outcome, hinted at in this work, is that bottom-heavy IMFs such as the Salpeter function might be ruled out by the comparison between stellar and lensing masses.

The correlation presented in this Letter help to reconcile the apparent discrepancy between the low dark matter content from dynamical estimates using planetary nebulae (Romanowsky et al. 2003) ${ }^{2}$ and the dark matter dominated galaxies suggested by X-ray data (Loewenstein \& Mushotzky 2002). Mass estimates of faint X-ray ellipticals, albeit challenging, will definitively test the observed correlation.

We would like to thank Nicola Napolitano for a very useful discussion and for providing his data and LCDM predictions.

\section{REFERENCES}

Trujillo, I., Burkert, A. \& Bell., E. F., 2004, ApJ, 600, L39

Binney, J. J., Dowrick, N. J., Fisher, A. J., \& Newman, M. E. J., 1992, The Theory of Critical Phenomena, Oxford

Bruzual, G. \& Charlot, S. 2003, MNRAS, 344, 1000

Chabrier, G., 2003, PASP, 115, 763

Dekel, A., et al., 2005, astro-ph/0501622

Falco, E. E., Gorenstein, M. V., Shapiro, I. I., ApJL, 289, 1

Ferreras, I. \& Silk, J., 2000, MNRAS, 316, 786

Fitzpatrick, E. L., 1999, PASP, 111, 63

Gorenstein, M. V., Falco, E. E., Shapiro, I. I., ApJ, 327, 693

Kroupa, P., Tout, C. A. \& Gilmore, G., 1993, MNRAS, 262, 545

Keeton, C. R., Kochanek, C. S., Falco, E. E. 1998, 509, 561

Keeton, C. R. \& Winn, J. N. 2003, ApJ, 590, 39

Kochanek, C. S., et al. 2000, ApJ, 543, 131

Koopmans, L. V. E., \& Treu, T. 2003, ApJ, 583, 60

Loewenstein, M. \& Mushotzky, R., 2002, astro-ph/0208090

Miller, G. E. \& Scalo, J. M., 1979, ApJS, 41, 513
Mobasher, B., et al. 1999, MNRAS, 304, 225

Napolitano, N. R. et al., 2005, MNRAS, 357, 691

Romanowsky, A. J., et al., 2003, Science, 301, 1696

Rusin, D. et al. 2003, ApJ, 587, 143

Saha, P. 2000, AJ, 120, 1654

Saha, P. 2003, Principles of Data Analysis, Cappella Archive.

Saha, P. \& Williams, L. L. R., 2004, AJ, 127, 2604

Salpeter, E. E., 1955, ApJ, 121, 161

Treu, T., \& Koopmans, L. V. E. 2002a, ApJ, 575, 87

Treu, T., \& Koopmans, L. V. E. 2002b, MNRAS, 337, L6

Trotter, C.S., Winn, J.N., Hewitt, J.N. 2000, ApJ, 535, 671

Spergel, D. N., et al., 2003, ApJS, 148, 175

Wechsler, R. H., Bullock, J. S., Primack, J. R., Kravtsov, A. V. \& Dekel, A., 2002, ApJ, 568, 52

Williams, L. L. R. \& Saha, P., 2000, ApJ, 119, 439

Wyse, R. F. G., 1998, in: Gilmore, G., Howell, D. (Eds.), The Stellar Initial Mass Function, ASP Conf. Ser., Vol. 142, ASP, San Francisco, CA, p. 89

\footnotetext{
${ }^{2}$ Recently Dekel et al. (2005) have argued that the assumption of isotropic PNe orbits made by Romanowsky et al. (2003) resulted in the mass being underestimated. However, our result that $\nabla \Upsilon$ is small for $\log \left(M_{s t} / M_{\odot}\right)=11-11.5$ suggests that while in general velocity anisotropy is an important effect, it is less so for a dynamically old population like PNe.
} 